PROFESSOR INGMAR BLUMCKE (Orcid ID : 0000-0001-8676-0788)

PROFESSOR THOMAS S JACQUES (Orcid ID : 0000-0002-7833-2158)

DR MARIA THOM (Orcid ID : 0000-0001-7712-2629)

Article type : Review

Corresponding author mail id : bluemcke@uk-erlangen.de

\title{
Challenges in the histopathological classification of ganglioglioma and DNT: microscopic agreement studies and a preliminary genotype-phenotype analysis
}

Ingmar Blümcke ${ }^{1,2}$, Roland Coras ${ }^{1}$, Annika K. Wefers ${ }^{3}$, David Capper ${ }^{4,15}$, Eleonora Aronica $^{5,6}$, Albert Becker ${ }^{7}$, Mrinalini Honavar ${ }^{8}$, Thomas J. Stone ${ }^{9}$, Thomas S. Jacques ${ }^{9}$, Hajime Miyata $^{10}$, Angelika Mühlebner ${ }^{6,11}$, Jose Pimentel ${ }^{12}$, Figen Söylemezoğlu ${ }^{13}$, Maria Thom $^{14}$

${ }^{1}$ Department of Neuropathology, University Hospital Erlangen, Germany

${ }^{2}$ Epilepsy Center, Cleveland Clinic, Ohio, USA

${ }^{3}$ Department of Neuropathology, Institute of Pathology, Ruprecht-Karls-University Heidelberg, Heidelberg, Germany

${ }^{4}$ Charité - Universitätsmedizin Berlin, corporate member of Freie Universität Berlin, Humboldt-Universität zu Berlin, and Berlin Institute of Health, Institute of Neuropathology, Berlin, Germany

${ }^{15}$ German Cancer Consortium (DKTK), Partner Site Berlin, German Cancer Research Center (DKFZ), Heidelberg, Germany

${ }^{5}$ Department of (Neuro)Pathology, Academic Medisch Centrum (AMC), Amsterdam, The Netherlands

${ }^{6}$ Stichting Epilepsie Instellingen Nederland (SEIN), The Netherlands

${ }^{7}$ Department of Neuropathology, University of Bonn Medical Centre, Bonn, Germany

This article has been accepted for publication and undergone full peer review but has not been through the copyediting, typesetting, pagination and proofreading process, which may lead to differences between this version and the Version of Record. Please cite this article as doi: 10.1111/nan.12522

This article is protected by copyright. All rights reserved. 
${ }^{8}$ Department of Anatomic Pathology, Hospital Pedro Hispano, Matosinhos, Portugal

${ }^{9}$ Developmental Biology and Cancer Section, UCL Great Ormond Street Institute of Child Health and the Department of Histopathology, Great Ormond Street Hospital for Children NHS Foundation Trust, London, United Kingdom

${ }^{10}$ Department of Neuropathology, Research Institute for Brain and Blood Vessels -AKITA, Akita, Japan

${ }^{11}$ Department of Pediatrics, Medical University Vienna, Vienna, Austria

${ }^{12}$ Laboratory of Neuropathology, Department of Neurology, Hospital de Santa Maria (CHLN), Lisbon, Portugal

${ }^{13}$ Department of Pathology, Faculty of Medicine, Hacettepe University, Ankara, Turkey

${ }^{14}$ Dept. of Neuropathology, Institute of Neurology, University College London, UK

Correspondence should be directed to:

Dr. Ingmar Blumcke, Department of Neuropathology, University Hospital Erlangen, Schwabachanlage 6, D - 91054 Germany

\section{Key words:}

epilepsy, seizure, neuropathology, brain tumour

\section{Abstract}

Low-grade epilepsy-associated brain tumours (LEAT) are the second most common cause for drug-resistant, focal epilepsy, i.e. ganglioglioma (GG) and dysembryoplastic neuroepithelial tumours (DNT). However, molecular pathogenesis, risk factors for malignant progression, and their frequent association with drug-resistant focal seizures remain poorly understood. This contrasts recent progress in understanding the molecular-genetic basis and targeted treatment options in diffuse gliomas. The Neuropathology Task Force of the International League against Epilepsy examined available literature to identify common obstacles in diagnosis and research of LEAT. Analysis of 10 published tumour series from epilepsy surgery pointed to poor interrater agreement for the histopathology diagnosis. The Task Force tested this hypothesis using a web-based microscopy agreement study. In a 
series of 30 LEAT, 25 raters from 18 countries agreed in only $40 \%$ of cases. Highest discordance in microscopic diagnosis occurred between GG and DNT variants, when oligodendroglial-like cell patterns prevail, or ganglion cells were difficult to discriminate from pre-existing neurons. Suggesting new terminology or major histopathological criteria did not satisfactorily increase the yield of histopathology agreement in 4 consecutive trials. To this end, the Task Force applied the WHO 2016 strategy of integrating phenotype analysis with molecular-genetic data obtained from panel sequencing and 450k methylation arrays. This strategy was helpful to distinguish DNT from GG variants in all cases. The Task Force recommends, therefore, to further develop diagnostic panels for the integration of phenotype-genotype analysis in order to reliably classify the spectrum of LEAT, carefully characterize clinically meaningful entities and make better use of published literature.

\section{Introduction}

Amongst the large histopathological spectrum of neoplastic lesions specified in the revised 4th edition of the WHO classification of tumours of the central nervous system [1], a variety of entities can be identified in patients with early onset of focal epilepsy before age 18 years (Table 1). These tumours have also been referred to as low-grade epilepsy-associated tumours (LEAT) [2-6]. Ganglioglioma (GG) and dysembryoplastic neuroepithelial tumours (DNT) are the most frequent LEAT entities. They comprise more than $80 \%$ of tumours herein grouped together as LEAT (Table 1) and $65 \%$ of all brain tumours encountered in large epilepsy surgery series[7]. However, they represent only $2-5 \%$ of tumours in the general cohort of neuro-oncology patients [8, 9]. From published series, GG and DNT share the following catalogue of histopathological and clinical features: (1) GG and DNT occur predominately in the temporal lobe (> 80\%). (2) GG and DNT provoke seizure onset in childhood (mean age at onset $=13$ years). (3) GG and DNT behave clinically in a benign manner and correspond mostly to WHO I . However, atypical and anaplastic features have been described in the literature. (4) GG and DNT have a histologically variable appearance with oligodendroglial or astrocytic appearing cells with or without a prominent dysplastic neuronal component. (5) GG and DNT are considered as tumours occurring during neurodevelopment and often associate with cortical dysplasia (FCD IIIb) [10]. (6) GG and DNT do not present genetic mutations or deletions typically observed in diffuse gliomas (IDH1, ATRX, 1p/19q co-deletion). In contrast, PI3K-AKT-mTOR and RAS-RAF-MAPK pathways are likely to be involved, i.e. BRAF V600E mutations are often encountered in $G G$, whereas DNT accumulate FGFR1 mutations or gene duplications [3, 11-17].

This article is protected by copyright. All rights reserved. 
The 2016 revised WHO classification has not introduced an integrated diagnostic genotypephenotype analysis for GG and DNT [3], and clinically meaningful molecular-pathological subgroups need yet to be established as recently exemplified with the description of polymorphous low-grade neuroepithelial tumour of the young with recurrent MAPK alterations [11]. Over-interpretation of the wide histopathology spectrum of LEAT remains challenging in daily routine neuropathology work-up [8]. LEAT resected in adult patients, presenting neuropathologically with IDH-1 wildtype and a predominant glial phenotype, will be at particular risk for over-interpretation into the group of malignant glioma [18]. Analysis of 4.454 tumours obtained from adults and children, published in 8 single-centre epilepsy surgery series, revealed huge differences in geographical prevalence (Table 2), ranging from $4 \%$ to $44 \%$ for glioma, $7 \%$ to $50 \%$ for GG, and $13 \%$ to $80 \%$ for DNT [6, 19-24]. Uncertainty is not restricted to the histological classification but also observed in tumour grading [25-28]. LEAT carry only remote risk for malignant progression, and the WHO classification did not define atypical variants for GG and DNT. Malignant tumour progression of GG and DNT has been reported, however, in few cases [29-31]. A reliable classification of LEAT is thus of paramount importance not only for histopathological routine diagnosis but also further patient management.

Taken together, available literature of LEAT assembled an ambiguous histopathology landscape, confounding cross-comparisons on molecular-genetic findings, immunohistochemical surrogates or clinically meaningful variants between published series $[3,8]$. Similar to the long-standing debate on oligoastrocytomas, which was finally solved by molecular genetic analysis [32, 33], we propose to work towards a biologically driven molecular classification for LEAT that can be used to identify histological, immunohistochemical and/or molecular parameters for specific diagnostic categorization. An international working group embedded into the ILAE Task Force of Neuropathology of the Diagnostic Methods commission has addressed these challenges and we describe herein a consensus report addressing areas of challenges and obstacles towards better understanding and diagnostic work-up of LEAT, in particular of GG and DNT variants.

This article is protected by copyright. All rights reserved. 


\section{Web-based virtual microscopy agreement studies}

Interrater agreement of LEAT was assessed using a collaborative virtual microscopy platform (CVMP) developed by Fraunhofer Society, Erlangen, Germany (www.cvmp.de) [34]. Thirty-eight neuropathologists from 19 countries were invited to review 30 de-identified tumours selected from the German Neuropathology Reference Center for Epilepsy Surgery in Erlangen, Germany. All patients suffered from drug-resistant epilepsy, but no clinical information was made available at time of microscopic review. A microscope scanner (Zeiss Z1, Oberkochen, Germany) was used to digitize HE stained slides, as well as GFAP-, MAP2-, NeuN-, CD34- and Ki-67-immunohistochemical reactions for all cases. A total of 180 digital scans were available for virtual microscopic review. Twenty-five participants completed the review within the requested 6-week time period. All reports were de-identified by the system's administrator (not member of the agreement study) and collected as exceldatasheet. We assigned agreement if $\geq 75 \%$ of raters achieved the same diagnosis for one patient. Twelve out of 30 tumour samples, 6 GG and 6 DNT, reached such agreement $(40 \%$, Table 3). Abbreviations were as following: $A A=$ anaplastic astrocytoma, $A G G=$ atypical ganglioglioma; AnaGG = anaplastic ganglioglioma; $\mathrm{ANET}=$ angiocentric glioma; $\mathrm{DA}=$ diffuse astrocytoma; DNT = dysembryoplastic neuroepithelial tumour; GC = gangliocytoma; $\mathrm{GG}=$ ganglioglioma; $\mathrm{GNT}=$ glio-neuronal tumour, not otherwise specified; $\mathrm{O}=$ oligodendroglioma; $\mathrm{OA}=$ mixed oligoastrocytoma; $\mathrm{PA}=$ pilocytic astrocytoma; $\mathrm{PGNT}=$ papillary glio-neuronal tumour; PXA = pleomorphic xanthoastrocytoma; RGNT = rosetted glio-neuronal tumour. Typical examples of agreed and not-agreed tumours were shown in Figure 1 - 3.

The $1^{\text {st }}$ LEAT agreement trial revealed four major areas in need of clarification. These areas are likely to also represent common obstacles for research and difficulties to reliably interpret published results. These challenges likely reflect also the different prevalence observed in our literature survey (Table 1).

\section{Challenges in the histopathological classification of DNT and GG}

\section{Nodular or diffuse growth patterns (Figures 1-2)}

Glio-neuronal tumours are regarded usually as hamartomatous mass lesions with nodular or cystic appearance and well demarcated from adjacent brain parenchyma. 
Indeed, many LEATs present with nodular growth patterns (Figure 2), located in neocortex or white matter, and composed of variable size ranging from several 100 $\mu \mathrm{m}$ to even $\mathrm{mm}$ scales, which make them visible on MRI. Other patterns include cysts or white matter rarefaction (Figure 1). Hence, DNT and GG also showing diffuse growth patterns are difficult-to-classify according to current standards. These include a diffuse and cell-sparse infiltration of the neocortex and white matter, subpial tumour growth or multiple tumour cell clusters remote from the mass lesion. A typical example representing such a tumour variant and which was provisionally termed herein diffuse glio-neuronal tumour (dGNT; see below) is depicted on Figure 1. The immunohistochemical marker CD34 is often helpful to reveal such patterns. When reviewing only H\&E stained sections those patterns may be difficult to distinguish from associated FCDIllb. In glio-neuronal tumours composed predominately of oligodendroglial cells, a diffuse subtype of DNT has been introduced to align this pattern to the WHO classification [19, 25]. Review of examples from our case series provoked controversial group discussion with the conclusion that this pattern remains difficult to attribute to either DNT or GG.

\section{Oligodendroglial or astrocytic cell components (Figure 3)}

The glial component in DNT and GG is predominating that of dysplastic neurons. Hence, the histopathological appearance of glial cells can be primarily oligodendroglial-like (Figure 3), astrocytic (Figure 1) or admix both phenotypic elements. One possible strategy in the diagnostic assessment of these patterns apparently was to favourably assign OLC predominance to the spectrum of DNT and astrocytic predominance to the spectrum of GG. In practise, however, glial patterns occur often simultaneously or vary regionally. Without access to anatomically intact large resections, the diagnostic decision may be compromised by tissue sampling. This discussion very much resembled that of mixed oligo-astrocytomas during past decades. Similar to the latter debate, our group could not agree on histopathology criteria approved by consecutive microscopic agreement studies (see below LEAT 1$5)$. 


\section{The ganglion cell component}

The concept of glio-neuronal tumours arose from early descriptions of bi-phasic differentiation patterns consisting of neurons and glial cell. Dysplastic neurons should be identified by the presence of multiple nuclei and later also by abnormal perimembraneous synaptophysin staining. In a gangliocytoma, these cells prevail without admixture of any neoplastic glial cell component. These concepts were difficult to verify microscopically and have never been scientifically proven. It is also believed, that glial cells underwent neoplastic transformation, which was partially supported by laser-microdissection experiments [35]. However, the post-mitotic nature of neurons and very low proliferation activity in most DNT and GG make it often difficult to establish any neoplastic process. With the concept of tumour origin from a founding stem cell, any bi-phasic appearance became biologically explicable, hence scientific confirmation of both lineages as being neoplastic remains pending. Another key question was how many dysplastic neurons need to be present to call a tumour GG or DNT. Again, sampling artefacts may challenge diagnostic decision, as clusters of abnormal neurons not otherwise explicable by anatomical localization may be focal and detectable only in large enough specimens. Review of examples from our case series provoked controversial group discussion with the conclusion that tumours with diffuse growth pattern and prominent CD34 immunoreactivity may or may not include dysplastic neurons and that this pattern attributed also to disagreement between $G G$ versus diffuse gliomas or PXA.

\section{Risk factors for malignant progression}

The 2016 WHO classification of tumours of the central nervous system did not promote grading of atypical $\left(\mathrm{WHO} \mathrm{II}^{\circ}\right)$ variants of DNT or GG. Hence, malignant progression of both tumour entities has been observed in clinical practice and described in published literature $[29,30,36]$. Notwithstanding, this issue is of utmost importance for clinical management and therapeutic counselling of a young patient with LEAT. Unfortunately, disagreement in the LEAT 1 trial affected WHO grading, and the group decided that this issue needs careful attention and should be addressed separately from terminology issues. 


\section{Efforts for better terminology use in microscopic examination of LEAT}

The Task Force tested different strategies to overcome the aforementioned problems in diagnostic consensus of microscopy-based tissue examination. The following list of strategies was chosen and tested in 4 consecutive web-based agreement studies (Table 3).

LEAT 2 (A-B-C classification yielding 47\% agreement): The second agreement study was designed to test the usefulness of prespecified immunohistochemical markers for the histopathological classification of LEAT, following an A-B-C classification proposal by Blumcke et al. in 2014 [5]. Same raters which completed LEAT1 were invited to review the same set of 30 tumours and immunohistochemcial stains (cases were displayed in random order). 20 raters responded in the requested time period of 6 weeks. We assigned agreement if $\geq 75 \%$ of raters achieved the same diagnosis for one patient. Fourteen out of 30 tumour samples reached such agreement (47\%).

LEAT 3 (introducing major and minor criteria for histopathology; yielding 56\% agreement): $\mathrm{A} 1^{\text {st }}$ face-to-face consensus meeting for microscopic review was organized in Amsterdam, November 2014. All participants from LEAT1 were invited and thirteen colleagues from 6 countries followed the invitation. Consensus obtained at the meeting was as following: 1. Minimal clinical information should be provided: age of patient at surgery; age of patient at seizure onset (if applicable); location of MRI-visible lesion. 2. A catalogue of major and minor histopathology criteria of LEAT was defined for further evaluation. 3. DNT diagnosis should be applied by its original (classic) definition, with diffuse and non-specific forms to be abandoned. 4. Difficult-to-classify tumour subtypes should be classified as separate entities (Table 3): Diffuse glio-neuronal tumour (dGNT): this neoplasm is best characterized by CD34 positive tumour cells with a vesicular nucleus, often diffuse infiltration pattern, but less evident neuronal component. Composite/complex glio-neuronal tumour (cGNT): tumours showed two or more distinct differentiation patterns, which were otherwise described in the WHO classification as ganglioglioma (GG), DNT, pleomorphic xanthoastrocytoma (PXA), or pilocytic astrocytoma (PA). Glio-neuronal tumours, not otherwise specified (GNT-NOS) are difficult-to-classify tumours neither classified by the new LEAT categories, nor by the WHO scheme. Fifteen cases were selected from the previous series and 154 digitized slides made available for virtual microscopy (HE, GFAP,

This article is protected by copyright. All rights reserved. 
MAP2, Synaptophysin, NeuN, EMA, IDH1, CD34, Ki-67). A set of questions asked to rate the importance of proposed criteria as well as immunohistochemical stains. Histopathological diagnosis was pre-defined and selected by single choice. Eleven participants from the Amsterdam meeting completed the survey within the requested 6-week time period. Interrater agreement of $>75 \%$ was obtained for 10 cases $(56 \%)$.

LEAT 4 (confirmation study using same test cases yielding $78 \%$ agreement): The list of major and minor criteria obtained from LEAT 3 was reviewed according to the rater's judgement. Criteria achieving more than $80 \%$ agreement were classified as "major", criteria with $50-80 \%$ agreement as "minor". The same set of slides was presented in random order and the same list of participants were invited to review all cases within 6 weeks. All raters were asked to use the list of major and minor histopathology criteria for their review. This survey achieved agreement in 14 cases (78\%).

LEAT 5 (confirmation study using new test cases yielding 55\% agreement): A second meeting was organized to discuss results of LEAT3 and LEAT4. The discussion raised controversial issues in need of further clarification: 1. High agreement in LEAT 4 may be the result of a learning curve, as LEAT 1 to 4 contained the same set of cases (although in random order). It was agreed to confirm results with a new set of tumours. 2. WHO's expected strategy to introduce an integrated phenotype-genotype approach for glioma and embryonal tumour classification ( $4^{\text {th }}$ edition) was yet not met by the group. 3 . The suggested panel of 7 immunohistochemical stains may not be affordable in every country. It was agreed to provide only 1 additional immunohistochemistry. CD34 was chosen to test its capability as surrogate marker for the LEAT classification. A total of 27 LEAT cases were collected. A 450k methylation profile was available in all cases (see below), but results were not displayed during virtual microscopy review. Ten participants completed the LEAT 5 survey in the requested 6-week time period. $>75 \%$ agreement for diagnosis was achieved in 6 DNT, 5 dGNT, 3 GG, 1 GNT-NOS (55\%, predefined diagnosis to be chosen by single choice; Table 2).

This article is protected by copyright. All rights reserved. 


\section{Summary of microscopy agreement studies}

Increase in interrater agreement from LEAT 1 to LEAT 4 likely resulted from familiarity of presented cases rather than improved criteria for histopathological classification. Despite the fact, that we introduced comprehensive definitions for classic and new entities as well as major and minor histopathology criteria, none of these solutions were proven sufficient when new tumour samples were included. These results reflected the well-recognized dilemma in the differential diagnosis of low-grade paediatric glial and glio-neuronal tumours and we have to anticipate an ever-increasing spectrum of presenting histopathology patterns in LEAT with respect to cellular composition, differentiation and growth patterns.

Difficulties to classify LEAT can be also recognized by a growing number of LEAT entities and variants published in scientific literature [11, 19, 37-43] as well as previous and current WHO classification systems (with lack of evidence for clinically meaningful subtypes). Introducing more LEAT entities and harmonizing criteria in this study, however, did not increase the diagnostic yield.

Immunohistochemistry was considered essential for the classification of LEAT but recommended panels are large and difficult to apply in every histopathology laboratory. Hence, consensus surrogate markers for histopathology diagnosis are not yet approved.

We concluded therefore, to evaluate the benefit of molecular data for the diagnostic neuropathological work-up of LEAT, as recently proposed by the WHO for diffuse glioma and embryonal brain tumours.

\section{Integration of molecular diagnostics}

DNA methylation profiling was used to identify molecular classes independent of histological evaluation in 20 cases diagnosed as GG, DNT or diffuse glio-neuronal tumours. 500ng DNA were extracted from formalin fixed and paraffin embedded tissue blocks and analysed using Illumina Infinium HumanMethylation450 BeadChip arrays (450k) as previously described [44]. Additional single gene analyses were performed for hotspot mutations of BRAF and FGFR1. Copy number profiles (calculated from methylation array data) were investigated for loss of CDKN2A. Unsupervised cluster analysis of DNA methylation data (MAD Euclidian ward) surprisingly revealed only two main clusters (Figure 4). Cluster 1 consisted of all DNT of this series $(n=5)$. Cluster two consisted of all cases of $G G(n=8)$ and all cases of diffuse glio-neuronal tumours $(n=7)$. Interestingly the two clusters also clearly segregated by their

This article is protected by copyright. All rights reserved. 
expression of CD34 and BRAF mutation status: In the GG/diffuse glio-neuronal tumour cluster all cases were positive for CD34. Furthermore, all 10 BRAF V600E mutations of this series were detected in the GG cluster evenly spread out between GG and the diffuse glioneuronal tumours. In contrast, in the DNT cluster 4 out of 5 DNT samples showed no CD34 immunoreactive tumour mass or satellite tumour cells. The last case of DNT revealed a diminutive cluster of CD34 immunoreactive satellite cells (not shown). In both clusters no CDKN2A deletions or FGFR1 hotspot mutations in exons 12 or 14 were observed. The latter was surprising to the group and would have requested further investigations in a prospectively based molecular-diagnostic scenario, i.e. searching for FGFR1 duplications or other abnormalities $[12,15,17,45]$.

A recently published study [12] suggested that by unsupervised analysis of RNA expression or DNA methylation there are two major groups of epilepsy-associated tumours. In order to compare the results of this ILAE LEAT study to the Stone et al. results, we clustered the DNA methylation results of the former along with the cases included in the latter study. The methylation data was read into the $\mathrm{R}$ bioinformatics environment using Minfi and normalised with the included subset-quantile within array normalisation (SWAN) method. Probes located on the $\mathrm{X}$ and $\mathrm{Y}$ chromosomes were excluded. In addition, probes located within $50 \mathrm{bp}$ of an SNP, probes known to cross-hybridise, and probes with a minor allele frequency $>5 \%$ were excluded. Consensus clustering of methylation data alongside cases from Stone et al. was carried out using the ConsensusClusterPlus package according to the Ward method. Cases were clustered intro two groups using the top 10,000 most variable CpGs across the combined cohort as determined by median absolute deviation. All of the cases defined as GG in the LEAT study clustered with the group 1 of the Stone paper and all the cases defined as DNT in the LEAT study clustered with group 2 of the Stone paper. This result suggests that the findings of the current histological study are in keeping with the molecular findings in the Stone study.

The data of our integrated analysis was discussed during a final group meeting at the European Neuropathology conference in June 2016. Major conclusions from this meeting were as following:

1. Tumours with diffuse growth patterns, a variable glio-neuronal phenotype and immunopositivity for CD34, i.e. proposed as diffuse glio-neuronal tumour by this Task Force, share molecular similarities with GG rather than DNT.

This article is protected by copyright. All rights reserved. 
2. BRAF V600E mutations were detected only in tumours immunoreactive for CD34 or agreed as GG, but in none of those tumours agreed as DNT, supporting the roadmap of an integrated genotype-phenotype diagnosis of LEAT.

3. Methylation profiling [12, 44] should be further explored scientifically for its reliability in classifying LEAT.

\section{Outlook}

Low-grade tumours with seizure onset in children and young adults as major neurological presentation remain a challenging issue in routine histopathological diagnosis, but also represent an important interface between clinical and basic research [16, 18, 46, 47]. In addition, the bi-phasic and variable glio-neuronal phenotype challenges traditional concepts of tumourigenesis. Early disease onset, benign clinical courses, variable histomorphological patterns, detection of the onco-fetal marker CD34 and involvement of the PI3K-AKT-mTOR and RAS-RAF-MAPK pathways point towards compromised developmental signalling rather than enhanced proliferation/apoptosis of genetically transformed tumour progenies (as reasoned in high-grade tumours). Availability of surgical epilepsy specimens help to directly address these intriguing issues using the rapidly growing armamentarium of morphological, immunohistochemical, molecular, genetic as well as electrophysiological techniques.

Molecular-genetic studies in LEAT need to also address predictive factors to identify cases with an increased risk of tumour recurrence or malignant transformation. The proportion of these patients is rather small in LEAT, but will help to improve clinical treatment strategies in patients with chronic focal epilepsies and brain tumours. This would also represent a unique scenario and environment for a prospective clinical trial, which are largely missing in the field of LEAT and epilepsy surgery. Given that the majority of these tumours are rare and grow slowly, multiple centres need to be included to recruit sufficient patient numbers within a reasonable time period. An additional goal should be to develop reference pathology centres and bio-repositories of surgical brain specimens and matched blood samples across continents to allow for a systematic molecular testing, keeping pace with new technologies or biomarkers as they become available.

This article is protected by copyright. All rights reserved. 
This report was written by experts selected by the International League Against Epilepsy (ILAE) and was approved for publication by the ILAE. Opinions expressed by the authors, however, do not necessarily represent the policy or position of the ILAE.

We confirm that we have read the Journal's position on issues involved in ethical publication and affirm that this report is consistent with those guidelines.

\section{Disclosure}

David Capper declares shared inventorship of a "DNA-methylation based method for classifying tumour species of the brain". A patent has been applied for this method (EP 3067432 A1). All terms are being managed by the German Cancer Research Center in accordance with its conflict of interest policies. All other authors report no conflict of interest in relation to this manuscript. The Editors of Neuropathology and Applied Neurobiology are committed to peer-review integrity and upholding the highest standards of review. As such, this article was peer-reviewed by independent, anonymous expert referees and the authors had no role in either the editorial decision or the handling of the paper.

\section{Acknowledgment}

We thank C. Münzenmayer, S.Friedl and T.Bindl from the Fraunhofer Institute for Integrated Circuits IIS, Erlangen, Germany for their continuous efforts to develop and maintain the CVMP platform. We kindly thank also Arie Perry (San Francisco, USA), Buge Oz (Istanbul, Turkey), Catherine Godfraind (Louvain-la-Neuve, Belgium), Fabio Rogerio (Campinas, Brazil), Felice Giangaspero (Rome, Italy), Brian Harding (Philadelphia, USA), Harvey Sarnat (Calgary, Canada), Josef Zamecnik (Prague, Czech Republic), Akiyoshi Kakita (Niigata, Japan), Nathalie Streichenberger (Lyon, France), Kim Se Hoon (Seoul, South Korea), and Piao Yue-Shan (Beijing, China) for their participation in the LEAT1 trial.

Our methylation studies were partially sponsored by the International League against Epilepsy (ILAE commission on diagnostic methods). IB and RC were supported by EU FP7 grant agreement \# 602531 DESIRE. EA was supported by KIKA (Stichting Kinderen Kankervrij, Stichting AMC Foundation and Stichting Knip. TJ was supported from Great 
Ormond Street Hospital Children's Charity, the Brain Tumour Charity, Children with Cancer and NIHR.

This report is independent research by the NIHR Biomedical Research Centre Funding Scheme (UK). The views expressed in this publication are those of the authors and not necessarily those of the NHS, the NIHR or the Department of Health (UK).

Table 1: WHO classification of 1846 low-grade epilepsy-associated brain tumours (LEAT) obtained from epilepsy surgery (table modified from [7])

\begin{tabular}{|c|c|c|c|c|}
\hline Tumour diagnosis & $\%$ & $\begin{array}{l}\text { Epilepsy } \\
\text { Onset }\end{array}$ & $\begin{array}{l}\text { Duration of } \\
\text { Epilepsy }\end{array}$ & $\begin{array}{l}\text { Location } \\
\text { in TL }\end{array}$ \\
\hline Ganglioglioma & $53.6 \%$ & 12.1 & 11.4 & $82 \%$ \\
\hline $\begin{array}{l}\text { Dysembryoplastic neuroepithelial } \\
\text { tumour }\end{array}$ & $30.6 \%$ & 14 & 12 & $68 \%$ \\
\hline Low grade tumour (NOS) & $5.9 \%$ & 14.6 & 11.5 & $82 \%$ \\
\hline Pilocytic astrocytoma & $5.4 \%$ & 14.1 & 12.3 & $71 \%$ \\
\hline Pleomorphic xanthoastrocytoma & $2.3 \%$ & 15 & 8 & $78 \%$ \\
\hline Isomorphic astrocytoma* & $0.9 \%$ & 16 & 11.4 & $41 \%$ \\
\hline Gangliocytoma & $0.9 \%$ & 12 & 17.1 & $87 \%$ \\
\hline Angiocentric glioma & $0.7 \%$ & 7.7 & 6.9 & $50 \%$ \\
\hline
\end{tabular}

Legend to Table 1: overview of histological tumour categories in a series of 1846 brain tumours obtained from epilepsy surgery and onset of focal seizures before age 18 years. NOS - not otherwise specified by referring neuropathologist. * Isomorphic astrocytoma was described in 2004 [39], but is not included in the WHO classification. Epilepsy onset and duration of epilepsy before surgery in years. TL $\%$ of tumours localized in the temporal lobe. 
Table 2: Survey of published tumour series obtained from epilepsy surgery

\begin{tabular}{|l|c|c|c|c}
\hline Series (n patients) & Glioma & DNT & GG & all GNT \\
\hline Kings London (92) & $7(8 \%)$ & $74(80 \%)$ & $6(7 \%)$ & $87 \%$ \\
\hline Grenoble (94) & $4(4 \%)$ & $61(65 \%)$ & $29(31 \%)$ & $95 \%$ \\
\hline UCL London (155) & $28(18 \%)$ & $88(56 \%)$ & $12(8 \%)$ & $79 \%$ \\
\hline Illinois (39) & $10(26 \%)$ & $10(26 \%)$ & $14(36 \%)$ & $62 \%$ \\
\hline Cleveland (218) & $26(20 \%)$ & $17(13 \%)$ & $48(37 \%)$ & $66 \%$ \\
\hline EEBB (2244) & $437(19 \%)$ & $565(25 \%)$ & $986(44 \%)$ & $75 \%$ \\
\hline Beijing (51) & $5(10 \%)$ & $10(20 \%)$ & $19(38 \%)$ & $82 \%$ \\
\hline Bonn (207) & $91(44 \%)$ & $29(14 \%)$ & $87(42 \%)$ & $56 \%$ \\
\hline Erlangen (1354) & $206(15 \%)$ & $246(18 \%)$ & $669(50 \%)$ & $72 \%$ \\
\hline & & & & \\
\hline
\end{tabular}

Legend to Table 2: Meta-analysis of 4285 tumours obtained from epilepsy surgery showed large variability of prevalence of DNT, GG and glioma. The group of glioma may include piloctic astrocytoma, pleomorphic xanthoastrocytoma, diffuse astrocytoma, oligodendroglioma, or angiocentric glioma of all WHO grades. GNT - all tumours with glioneuronal phenotype. EEBB - European Epilepsy Brain Bank. Modified from [6].

This article is protected by copyright. All rights reserved. 
Table 3: Summary of 5 LEAT agreement studies using a CVMP platform

\begin{tabular}{|c|c|c|}
\hline $\begin{array}{l}\text { Study } \\
\text { (year) }\end{array}$ & $\begin{array}{c}\text { Participants } \\
\text { (invited/completed) }\end{array}$ & Cases without agreement \\
\hline $\begin{array}{l}\text { LEAT } 1 \\
(2013)\end{array}$ & $\begin{array}{l}38 / 25 \text { reviewer } \\
30 \text { cases } \\
180 \text { slides } \\
40 \% \text { agreement }\end{array}$ & $\begin{array}{c}\text { Case 3: PGNT=9; GG=8; DNT=4; Case 5: } \\
\text { DNT=13; GG=4; } \mathrm{O}=2 ; \text { Case 8: } \mathrm{O}=8 ; \mathrm{OA}=5, \\
\text { DNT=5; Case 9: GG=15, GC=3, PXA=2, } \mathrm{O}=1 ; \\
\text { Case 10: GG=15;GC=9;AA=1; Case 11: } \\
\text { GG=18, DNT=5, DA=2, Case 13: GG=13, } \\
\text { DNT=8, AGG=1; Case 14: DNT=14, RGNT=2, } \\
\text { O=2, PGNT=2; Case 15: DNT=14, GG=6, PA=2; } \\
\text { Case 16: GG=7, DNT=7, AGG=6, DA=2; Case } \\
\text { 17: GG=15, DNT=9, DA=1; Case 18: DNT=17, } \\
\text { O=5, GG=1; Case 20: GG=13, PXA=6, AGG=2; } \\
\text { Case 25: DNT=16, GG=4, ANET=2; Case 26: } \\
\text { GG=12, PXA=5; PA=2 AGG=1, AnaGG=1; Case } \\
\text { 28: DNT=9, GG=8; DA=2, } O=2 ; \text { Case 29: GG=14, } \\
\text { DNT=6, DA=3; Case 30: DNT=15, GG=4, DA=2. }\end{array}$ \\
\hline $\begin{array}{c}\text { LEAT } 2 \\
(2014)\end{array}$ & $\begin{array}{l}25 / 20 \text { reviewer } \\
30 \text { cases } \\
180 \text { slides } \\
47 \% \text { agreement }\end{array}$ & $\begin{array}{l}\text { Case 1: BNET=13, GNET=3, CNET=2; Case 5: } \\
\text { GNET=8; DNET=6, ENET=2; Case 7: CNET=11, } \\
\text { DNET=7, BNET=2; Case 8: ENET=8, DNET=3, } \\
\text { GNET=4; Case 9: BNET=12, GNET=4, CNET=3; } \\
\text { Case 10: BNET=13, GNET=4, CNET=2; Case } \\
\text { 14: ENET=5, DNET=4, GNET=4; Case 15: } \\
\text { DNET=9, GNET=5, ANET=3; Case 17: } \\
\text { BNET=12, CNET=7, GG=1; Case 18: DNET=14, } \\
\text { CNET=2, ENET=2; Case 20: } \mathrm{BNET}=11, \\
\text { GNET=3, CNET=2; Case 22: GNET=12, } \\
\text { ENET=4, DNET=2; Case 25: DNET=7, CNET=4, } \\
\text { ENET=2, GNET=1; Case 26: BNET=13, } \\
\text { CNET=3, ANET=2; Case 28: GNET=7, DNET=4, } \\
\text { ENET=4, CNET=3; Case 30: DNET=5, INET=5, } \\
\text { CNET=3. }\end{array}$ \\
\hline $\begin{array}{l}\text { LEAT } 3 \\
(2015)\end{array}$ & $\begin{array}{l}13 / 11 \text { reviewer } \\
18 \text { cases } \\
154 \text { slides } \\
56 \% \text { agreement }\end{array}$ & $\begin{array}{l}\text { Case 3: } \mathrm{O}=7, \mathrm{OA}=2, \mathrm{DA}=2 \text {; Case } 5: \mathrm{PXA}=5 \text {, } \\
\mathrm{GG}=2, \mathrm{GNT}-\mathrm{NOS}=2 ; \text { Case } 8: \mathrm{DNT}=5, \mathrm{GNT}- \\
\mathrm{NOS}=4, \mathrm{dGNT}=1, \mathrm{OA}=1 ; \text { Case 11: } \mathrm{DNT}=6 \text {, } \\
\mathrm{dGNT}=3, \mathrm{cGNT}=2 ; \text { Case 12: } \mathrm{dGNT}=8, \mathrm{GG}=2 \text {, }\end{array}$ \\
\hline
\end{tabular}




\begin{tabular}{|c|c|c|}
\hline & & $\begin{array}{c}\text { GNT-NOS=1; Case 13: } \mathrm{DNT}=4, \mathrm{GNT}-\mathrm{NOS}=3, \\
\mathrm{iA}=3, \mathrm{dGNT}=1 ; \text { Case 17: } \mathrm{dGNT}=7, \mathrm{cGNT}=3, \\
\mathrm{GG}=1 \text {; Case 18: } \mathrm{GG}=7, \mathrm{GNT}-\mathrm{NOS}=2, \mathrm{cGNT}=1, \\
\mathrm{dGNT}=1\end{array}$ \\
\hline $\begin{array}{l}\text { LEAT } 4 \\
\text { (2015) }\end{array}$ & $\begin{array}{l}\text { 13/10 reviewer } \\
18 \text { cases } \\
154 \text { slides } \\
78 \% \text { agreement }\end{array}$ & $\begin{array}{c}\text { Case 5: } G G=4 \text {, no } D X=3, d G N T=2 ; c G N T=1 ; \\
\text { Case 7: } G G=7, d G N T=3 ; \text { Case 13: } D N T=4, \\
\text { GNT-NOS=3, iA=3; Case 18: } G G=7, c G N T=2, \\
d G N T=1\end{array}$ \\
\hline LEAT 5 & $\begin{array}{c}13 / 10 \text { reviewer } \\
27 \text { cases } \\
54 \text { slides } \\
55 \% \text { agreement }\end{array}$ & 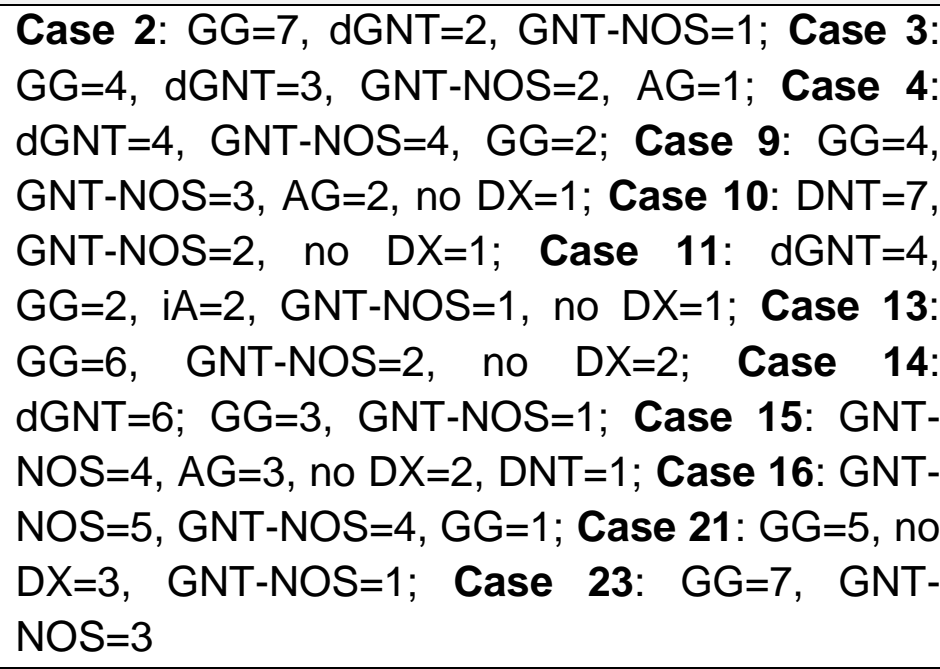 \\
\hline
\end{tabular}

Legend to Table 3: Note that LEAT 1 to LEAT4 presented with same cases in different number, order and access to additional immunohistochemistry. Reviewer: invited to the trial/completed the trial. LEAT 2 tested the ABC proposal only. LEAT 5 included new cases. See text for further information. The majority of non-agreed diagnosis ( $\mathrm{k}=<75 \%$ ) dealt with the spectrum of DNT and GG (73\% in LEAT1; $70 \%$ in LEAT3 and $81 \%$ in LEAT5). Abbreviations: $A G$ = angiocentric glioma; $A N E T$ - angiocentric neuroepithelial tumour, BNET - basic (CD34+) neuroepithelial tumour, cGNT = complex glio-neuronal tumour; CNET complex neuroepithelial tumour, DA = diffuse astrocytoma; dGNT = diffuse glio-neuronal tumour; $\mathrm{DN}(\mathrm{E}) \mathrm{T}$ = dysembryoplastic neuroepithelial tumour; ENET - epilepsy-associated neuroepithelial tumour not otherwise specified, GC = gangliocytoma; GG = ganglioglioma; GNT-NOS = glio-neuronal tumour, not otherwise specified; GNET - ganglion cell predominate neuroepithelial tumour, INET - isomorphic neuroepithelial tumour. $\mathrm{iA}=$ isomorphic astrocytoma; no $\mathrm{DX}=$ no diagnosis specified; $O=$ oligodendroglioma; $O A=$ mixed oligoastrocytoma; PXA = pleomorphic xanthoastrocytoma.

This article is protected by copyright. All rights reserved. 


\section{Figure 1: CD34 positive tumour with good interrater agreement in LEAT 5}
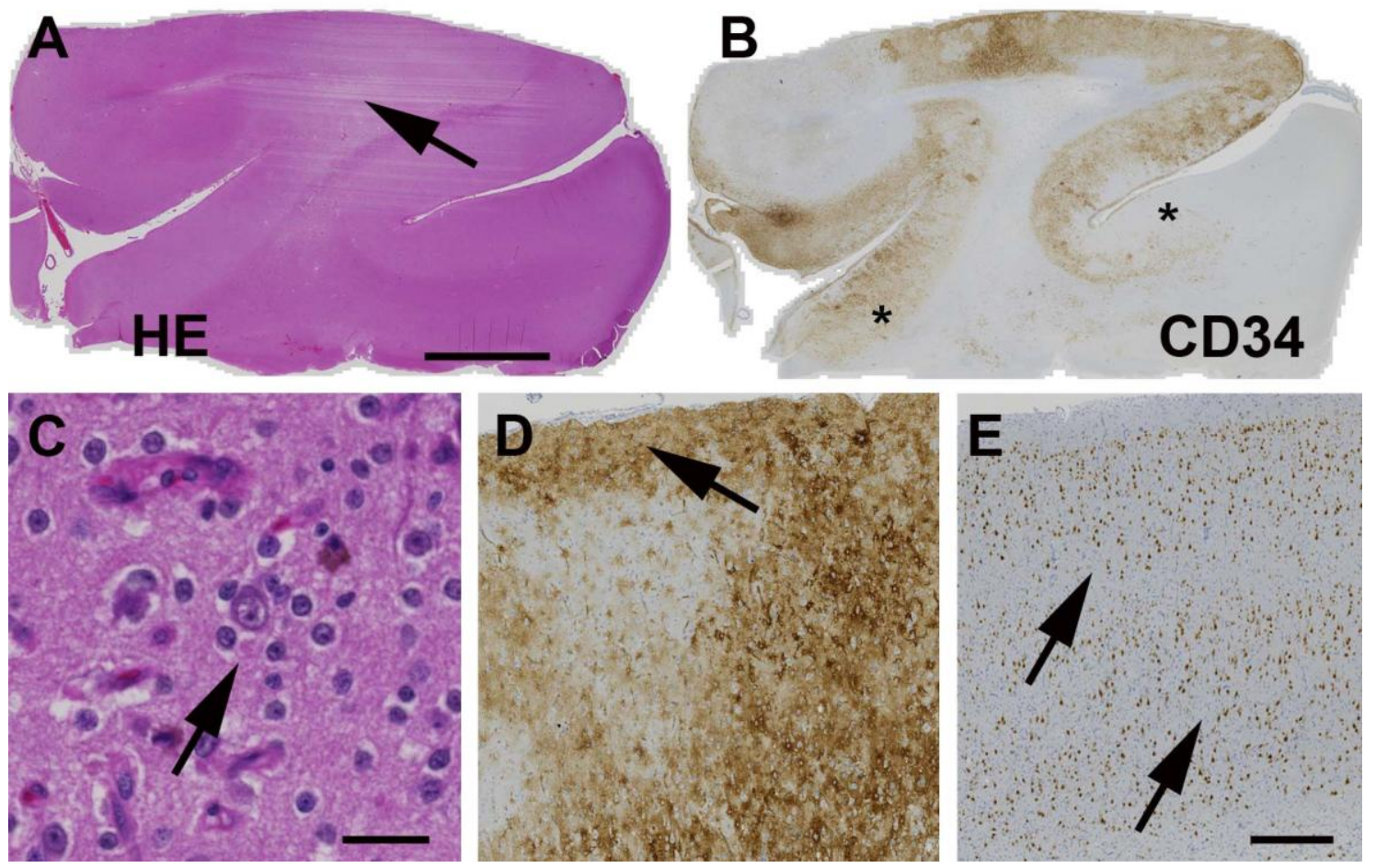

Legend to Figure 1: 31 year old male patient with right temporal lobe epilepsy starting at the age of 11 years (case \#17 in LEAT 5). A: HE staining (WSI) revealed a glio-neuronal tumour with diffuse infiltration and white matter rarefaction (arrow) B: WSI of CD34 immunohistochemistry confirmed diffuse infiltration of CD34 positive cells across sulci (asterisks) not readily visible on $\mathrm{HE}$ staining. Scale bar in $\mathrm{A}=2 \mathrm{~mm}$, applies also to $\mathrm{B}$. C: higher power magnification of tumour cell infiltrates. The small cell component often showed vesicular nuclei, whereas neurons (arrow) were difficult to distinguish from a pre-existing cell population. Scale bar $=50 \mu \mathrm{m}$. D: low power magnification of diffuse infiltration of CD34 positive cells in cortex up to layer 1 (arrow). E: Pre-existing neocortex with 6-layered architecture. Architectural abnormalities (arrows) resulted from infiltrating tumour (see adjacent section stained for CD34 in D), rather than being FCD Illb. NeuN immunohistochemistry. Scale bar $=200 \mu \mathrm{m}$, applies also to D. This tumour was classified as diffuse glio-neuronal tumour by all reviewers. Molecular classification: LGG-GG, BRAF V600E, CDKN2A balanced, FGFR1 wt (exons 12 and 14).

This article is protected by copyright. All rights reserved. 
Figure 2: CD34 positive tumour with poor interrater agreement LEAT5

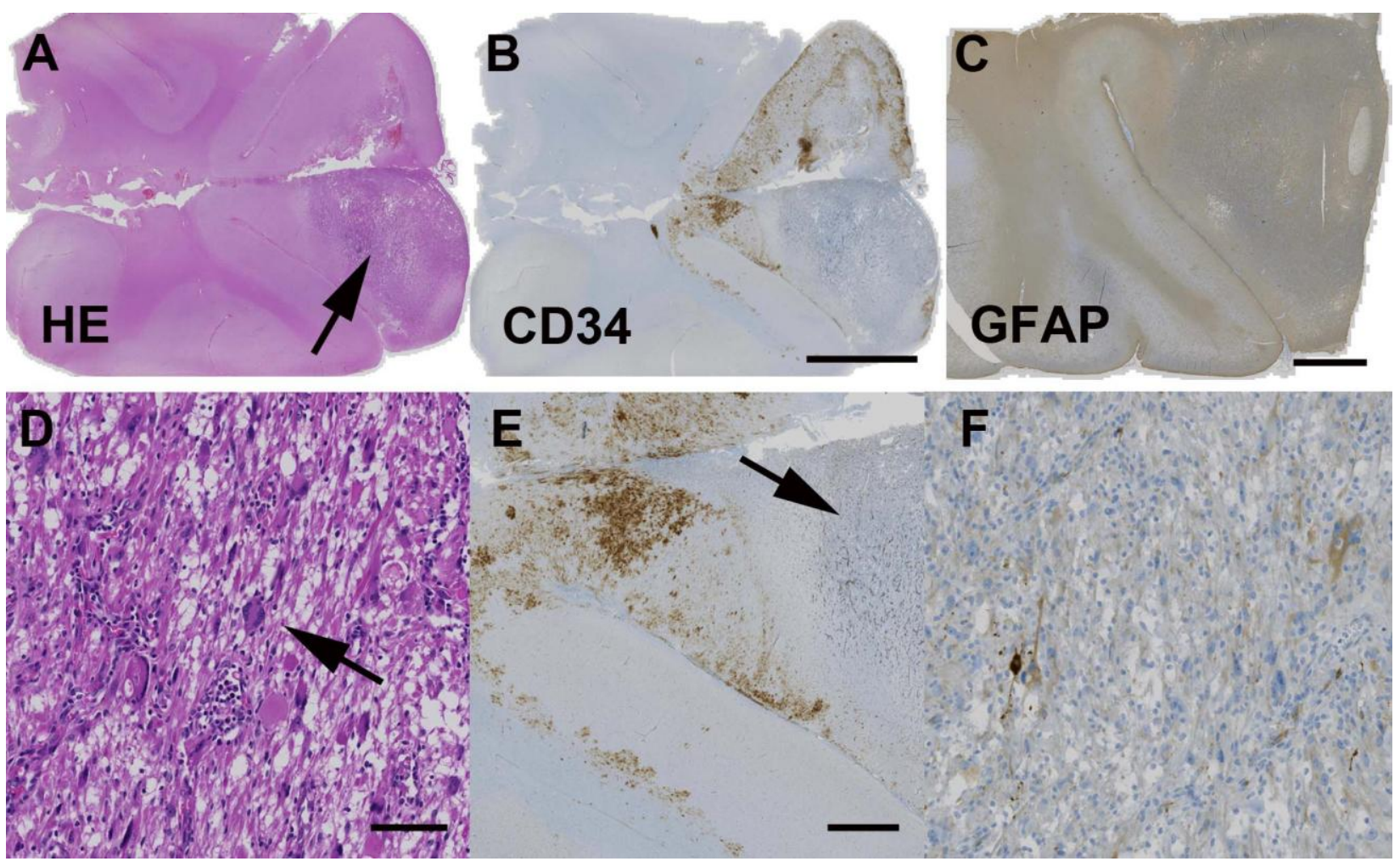

Legend to Figure 2: 32 year old female patient with temporal lobe epilepsy starting at the age of 28 years. A: WSI of HE staining with a nodular growth pattern (arrow). B: CD34immunohistochemistry of adjacent section showed diffuse and nodular tumour growth with different CD34 immunoreactivity patterns. Scale bar $=2 \mathrm{~mm}$, applies also to A. C: Dense GFAP staining could be observed in the tumour nodule. Scale bar $=1 \mathrm{~mm}$. D: High power magnification of the tumour nodule revealed multinucleated cells (arrow), eosinophic bodies and a pilocytic matrix (HE staining). E: The tumour nodule was not CD34-immunopositive (arrow), compared to diffuse infiltration patterns in adjacent neocortex. F: NeuN immunoreactivity in few dysplastic neuronal cell elements of the tumour nodule. Scale bar in $D=100 \mu \mathrm{m}$, applies also to $\mathrm{F}$. Scale bar in $E=500 \mu \mathrm{m}$. The following diagnoses were obtained from our reviewers: 4 × GG, $3 \times$ GNT-NOS, $2 \times$ AG, $1 \times$ no DX. Molecular classification: LGG-GG, BRAF V600E mutation, CDKN2A balanced, FGFR1 wt.

This article is protected by copyright. All rights reserved. 
Figure 3: CD34 negative DNT with very good interrater agreement in LEAT5

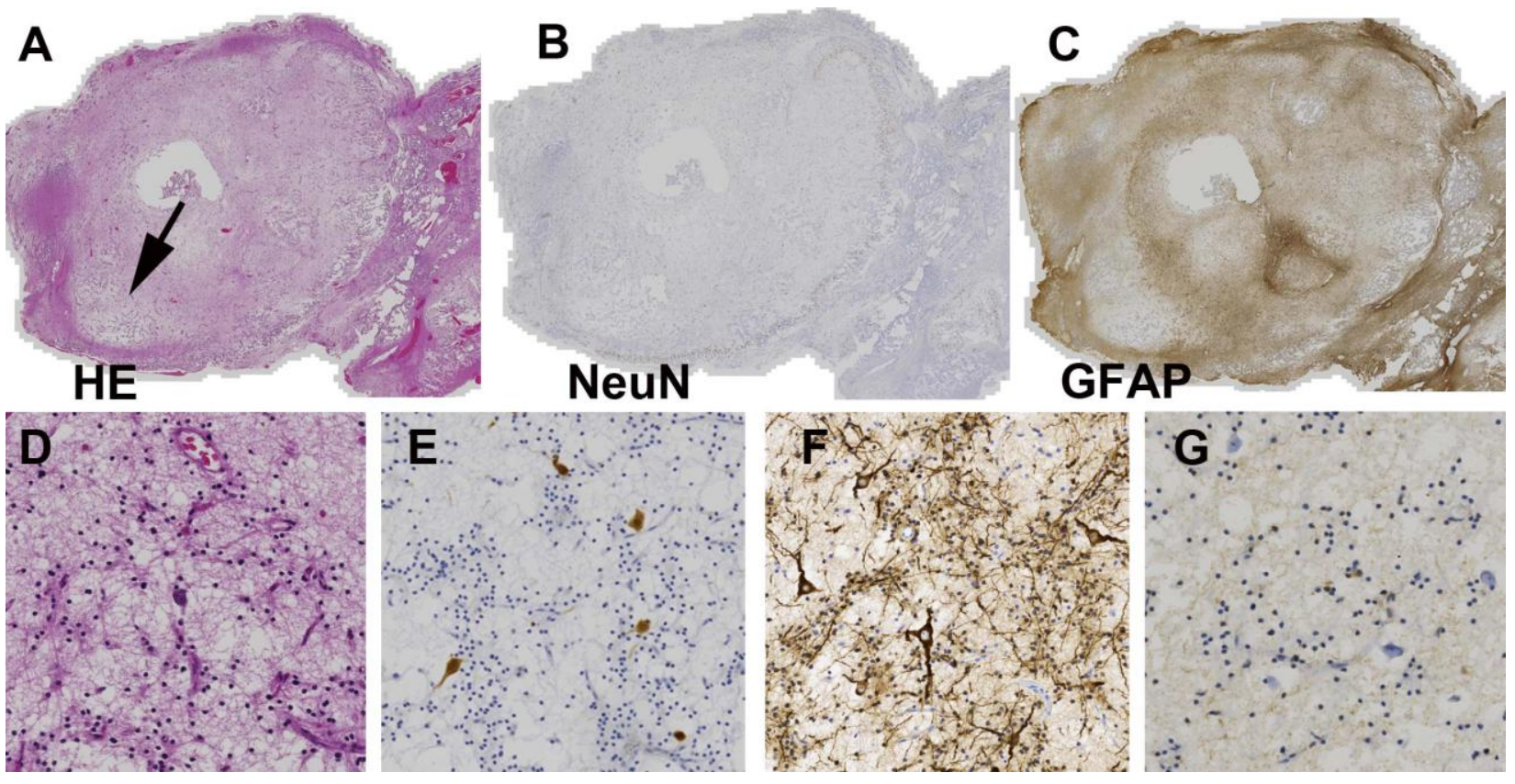

Legend to Figure 3: 11-years old patient with right temporal lobe epilepsy starting at the age of 1 year. A-C: whole slide digital imaging (WSI) showing a multinodular tumour. Arrow indicates area taken for magnifications shown in D-G. HE staining. D-E: specific glioneuronal element with floating neurons $(E=N e u N)$. F: MAP2 staining showing floating neurons and immunoreactive OLC. G: OLC were usually not GFAP-immunoreactive. Other phenotypic markers: IDH1 R132H negative, Ki-67<1\%, CD34 negative. The following diagnoses were obtained from our reviewers: 8 x DNT, 1 x ANET, 1 x no DX. Molecular classification: LGG-DNT, BRAF wt, CDKN2A balanced, FGFR1 wt.

This article is protected by copyright. All rights reserved. 
Figure 4: Unsupervised hierarchical cluster analysis of DNT, GG and diffuse glioneuronal tumours indicated molecular separation of two classes only

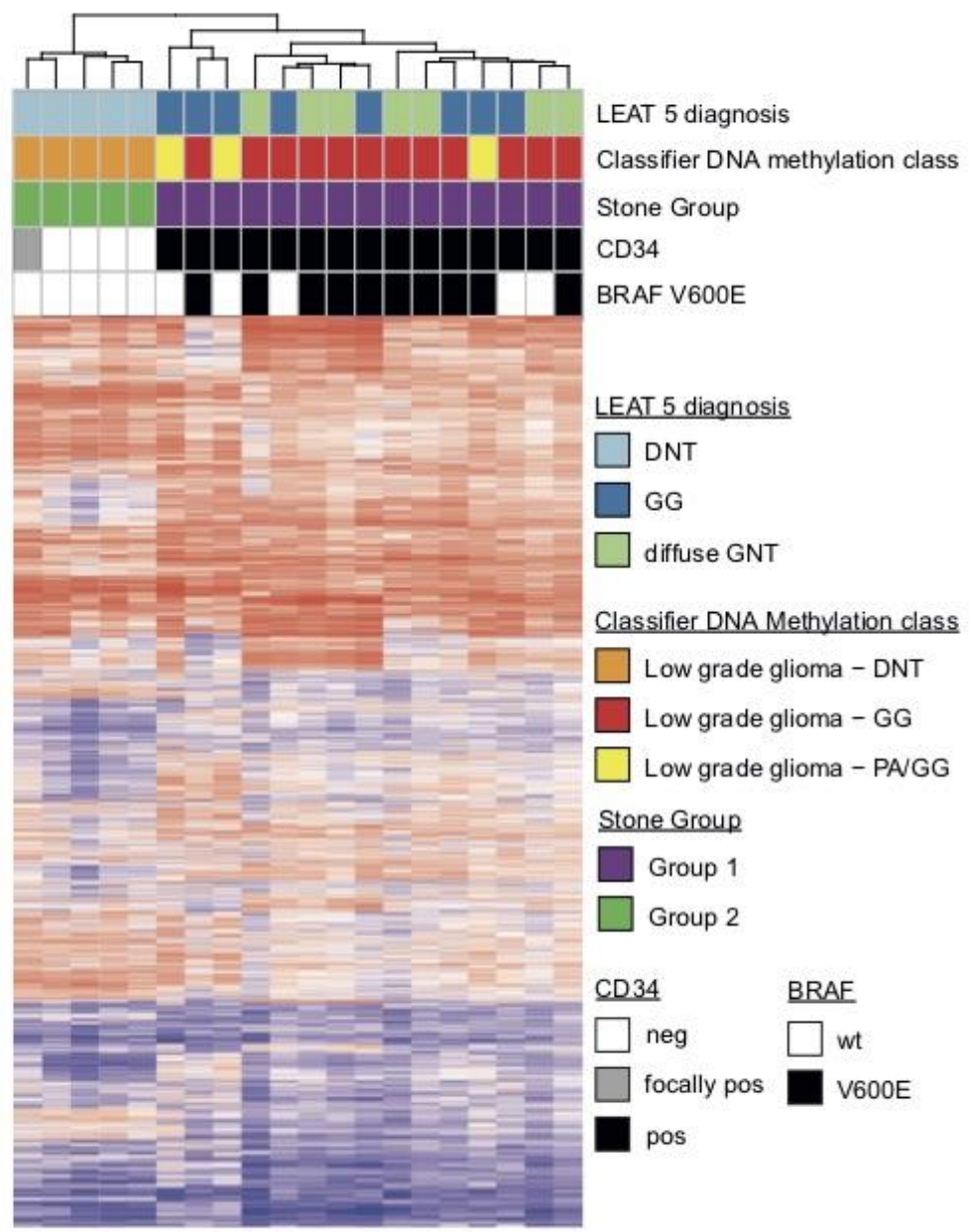

Legend to Figure 4: unsupervised hierarchical cluster analysis of 5 DNT, 8 GG and 7 diffuse glio-neuronal tumours indicated two main clusters. One cluster consisted of all cases of DNT, did not harbour BRAF mutations and was CD34 negative except for one case with a small cluster of positive satellite cells and clustered with group 2 tumours of the Stone et al. study. The other cluster was composed of all GG and diffuse glio-neuronal tumours, with a high rate of BRAF V600E mutations and CD34 immunoreactivity. These tumours clustered with group 1 tumours of the Stone et al. study. No FGFR1 hotspot mutations or CDKN2A deletions were observed in the series.

This article is protected by copyright. All rights reserved. 


\section{References}

1 Louis DN, Ohgaki H, Wiestler OD, Cavenee WK, Ellison DW, Figarella-Branger D, et al. WHO Classification of Tumours of the Central Nervous System (revised 5th edition). Lyon: IARC. 2016

2 Luyken C, Blumcke I, Fimmers R, Urbach H, Elger CE, Wiestler OD, et al The spectrum of longterm epilepsy-associated tumors: long-term seizure and tumor outcome and neurosurgical aspects. Epilepsia 2003 Jun; 44: 822-30

3 Blumcke I, Aronica E, Becker A, Capper D, Coras R, Honavar M, et al. Low-grade epilepsyassociated neuroepithelial tumours - the 2016 WHO classification. Nature reviews Neurology 2016 Dec; 12: 732-40

4 Blumcke I, Wiestler OD. Gangliogliomas: an intriguing tumor entity associated with focal epilepsies. Journal of neuropathology and experimental neurology $2002 \mathrm{Jul}$; 61: 575-84

5 Blumcke I, Aronica E, Urbach H, Alexopoulos A, Gonzalez-Martinez JA. A neuropathologybased approach to epilepsy surgery in brain tumors and proposal for a new terminology use for longterm epilepsy-associated brain tumors. Acta neuropathologica 2014 Jul; 128: 39-54

6 Thom M, Blumcke I, Aronica E. Long-term epilepsy-associated tumors. Brain Pathol 2012 May; 22: 350-79

7 Blumcke I, Spreafico R, Haaker G, Coras R, Kobow K, Bien CG, et al. Histopathological Findings in Brain Tissue Obtained during Epilepsy Surgery. The New England journal of medicine 2017 Oct 26; 377: 1648-56

8 Holthausen $\mathrm{H}$, Blumcke I. Epilepsy-associated tumours: what epileptologists should know about neuropathology, terminology, and classification systems. Epileptic disorders : international epilepsy journal with videotape 2016 Sep 1; 18: 240-51

9 Jemal A, Siegel R, Ward E, Murray T, Xu J, Smigal C, et al. Cancer statistics, 2006. CA: a cancer journal for clinicians 2006 Mar-Apr; 56: 106-30

10 Blumcke I, Thom M, Aronica E, Armstrong DD, Vinters HV, Palmini A, et al. The clinicopathological spectrum of Focal Cortical Dysplasias: a consensus classification proposed by an ad hoc Task Force of the ILAE Diagnostic Methods Commission. Epilepsia 2011; 52: 158-74

11 Huse JT, Snuderl M, Jones DT, Brathwaite CD, Altman N, Lavi E, et al. Polymorphous lowgrade neuroepithelial tumor of the young (PLNTY): an epileptogenic neoplasm with oligodendroglioma-like components, aberrant CD34 expression, and genetic alterations involving the MAP kinase pathway. Acta neuropathologica 2016 Nov 3; 133: 417-29

12 Stone TJ, Keeley A, Virasami A, Harkness W, Tisdall M, Izquierdo Delgado E, et al. Comprehensive molecular characterisation of epilepsy-associated glioneuronal tumours. Acta neuropathologica 2018 Oct 20; 135: 115-29

This article is protected by copyright. All rights reserved. 
13 Koelsche C, Wohrer A, Jeibmann A, Schittenhelm J, Schindler G, Preusser M, et al. Mutant BRAF V600E protein in ganglioglioma is predominantly expressed by neuronal tumor cells. Acta neuropathologica 2013 Jun; 125: 891-900

14 Schindler G, Capper D, Meyer J, Janzarik W, Omran H, Herold-Mende C, et al. Analysis of BRAF V600E mutation in 1,320 nervous system tumors reveals high mutation frequencies in pleomorphic xanthoastrocytoma, ganglioglioma and extra-cerebellar pilocytic astrocytoma. Acta neuropathologica 2011 Mar; 121: 397-405

15 Fina F, Barets D, Colin C, Bouvier C, Padovani L, Nanni-Metellus I, et al. Droplet digital PCR is a powerful technique to demonstrate frequent FGFR1 duplication in dysembryoplastic neuroepithelial tumors. Oncotarget 2016 Oct 25:

16 Zhang J, Wu G, Miller CP, Tatevossian RG, Dalton JD, Tang B, et al. Whole-genome sequencing identifies genetic alterations in pediatric low-grade gliomas. Nat Genet 2013 Jun; 45: $602-12$

17 Rivera B, Gayden T, Carrot-Zhang J, Nadaf J, Boshari T, Faury D, et al. Germline and somatic FGFR1 abnormalities in dysembryoplastic neuroepithelial tumors. Acta neuropathologica 2016 Feb 26:

18 Brat DJ, Verhaak RG, Aldape KD, Yung WK, Salama SR, Cooper LA, et al. Comprehensive, Integrative Genomic Analysis of Diffuse Lower-Grade Gliomas. The New England journal of medicine 2015 Jun 25; 372: 2481-98

19 Honavar M, Janota I, Polkey CE. Histological heterogeneity of dysembryoplastic neuroepithelial tumour: identification and differential diagnosis in a series of 74 cases. Histopathology 1999 Apr; 34: 342-56

20 Pasquier B, Peoc HM, Fabre-Bocquentin B, Bensaadi L, Pasquier D, Hoffmann D, et al. Surgical pathology of drug-resistant partial epilepsy. A 10-year-experience with a series of 327 consecutive resections. Epileptic disorders : international epilepsy journal with videotape 2002 Jun; 4: 99-119

21 Prayson RA. Tumours arising in the setting of paediatric chronic epilepsy. Pathology 2010; 42: 426-31

22 Prayson RA. Brain tumors in adults with medically intractable epilepsy. Am J Clin Pathol 2011 Oct; 136: 557-63

23 Piao YS, Lu DH, Chen L, Liu J, Wang W, Liu L, et al. Neuropathological findings in intractable epilepsy: 435 Chinese cases. Brain Pathol 2010 Sep; 20: 902-8

24 Ruban D, Byrne RW, Kanner A, Smith M, Cochran EJ, Roh D, et al. Chronic epilepsy associated with temporal tumors: long-term surgical outcome. Neurosurgical focus 2009 Aug; 27: E6

25 Daumas-Duport C, Varlet P, Bacha S, Beuvon F, Cervera-Pierot P, Chodkiewicz JP. Dysembryoplastic neuroepithelial tumors: nonspecific histological forms -- a study of 40 cases. Journal of neuro-oncology $1999 \mathrm{Feb}$; 41: 267-80

This article is protected by copyright. All rights reserved. 
26 Campos AR, Clusmann H, von Lehe M, Niehusmann P, Becker AJ, Schramm J, et al. Simple and complex dysembryoplastic neuroepithelial tumors (DNT) variants: clinical profile, MRI, and histopathology. Neuroradiology 2009 Jul; 51: 433-43

27 Dozza DC, Rodrigues FF, Chimelli L. Dysembryoplastic neuroepithelial tumor originally diagnosed as astrocytoma and oligodendroglioma. Arquivos de neuro-psiquiatria 2012 Sep; 70: 710-4

28 Chassoux F, Landre E, Mellerio C, Laschet J, Devaux B, Daumas-Duport C. Dysembryoplastic neuroepithelial tumors: epileptogenicity related to histologic subtypes. Clinical neurophysiology : official journal of the International Federation of Clinical Neurophysiology 2013 Jun; 124: 1068-78

29 Majores M, von Lehe M, Fassunke J, Schramm J, Becker AJ, Simon M. Tumor recurrence and malignant progression of gangliogliomas. Cancer 2008 Dec 15; 113: 3355-63

30 Heiland DH, Staszewski O, Hirsch M, Masalha W, Franco P, Grauvogel J, et al. Malignant Transformation of a Dysembryoplastic Neuroepithelial Tumor (DNET) Characterized by GenomeWide Methylation Analysis. Journal of neuropathology and experimental neurology 2016 Apr; 75: 358-65

31 Ray WZ, Blackburn SL, Casavilca-Zambrano S, Barrionuevo C, Orrego JE, Heinicke H, et al. Clinicopathologic features of recurrent dysembryoplastic neuroepithelial tumor and rare malignant transformation: a report of 5 cases and review of the literature. Journal of neuro-oncology 2009 Mar 8; $94: 283-92$

32 Louis DN, Perry A, Reifenberger G, von Deimling A, Figarella-Branger D, Cavenee WK, et al. The 2016 World Health Organization Classification of Tumors of the Central Nervous System: a summary. Acta neuropathologica 2016 Jun; 131: 803-20

33 Sahm F, Reuss D, Koelsche C, Capper D, Schittenhelm J, Heim S, et al. Farewell to oligoastrocytoma: in situ molecular genetics favor classification as either oligodendroglioma or astrocytoma. Acta neuropathologica 2014 Oct; 128: 551-9

34 Coras R, de Boer OJ, Armstrong D, Becker A, Jacques TS, Miyata H, et al. Good interobserver and intraobserver agreement in the evaluation of the new ILAE classification of focal cortical dysplasias. Epilepsia 2012 Aug; 53: 1341-8

35 Becker AJ, Lobach M, Klein H, Normann S, Nothen MM, von Deimling A, et al. Mutational analysis of TSC1 and TSC2 genes in gangliogliomas. Neuropathology and applied neurobiology 2001 Apr; 27: 105-14

36 Luyken C, Blumcke I, Fimmers R, Urbach H, Wiestler OD, Schramm J. Supratentorial gangliogliomas: histopathologic grading and tumor recurrence in 184 patients with a median followup of 8 years. Cancer 2004 Jul 1; 101: 146-55

37 Schramm J, Luyken C, Urbach H, Fimmers R, Blumcke I. Evidence for a clinically distinct new subtype of grade II astrocytomas in patients with long-term epilepsy. Neurosurgery 2004 Aug; 55: 340-7; discussion 7-8

This article is protected by copyright. All rights reserved. 
38 Huse JT, Edgar M, Halliday J, Mikolaenko I, Lavi E, Rosenblum MK. Multinodular and vacuolating neuronal tumors of the cerebrum: 10 cases of a distinctive seizure-associated lesion. Brain Pathol 2013 Sep; 23: 515-24

39 Blumcke I, Luyken C, Urbach H, Schramm J, Wiestler OD. An isomorphic subtype of long-term epilepsy-associated astrocytomas associated with benign prognosis. Acta neuropathologica 2004 May; 107: 381-8

40 Schurr J, Coras R, Rossler K, Pieper T, Kudernatsch M, Holthausen H, et al. Mild malformation of cortical development with oligodendroglial hyperplasia in frontal lobe epilepsy: A new clinicopathological entity. Brain Pathol 2016 Jan 7; (epub ahead of print):

41 Lellouch-Tubiana A, Boddaert N, Bourgeois M, Fohlen $M$, Jouvet A, Delalande $O$, et al. Angiocentric neuroepithelial tumor (ANET): a new epilepsy-related clinicopathological entity with distinctive MRI. Brain Pathol 2005; 15: 281-6

42 Wang M, Tihan T, Rojiani AM, Bodhireddy SR, Prayson RA, lacuone JJ, et al. Monomorphous angiocentric glioma: a distinctive epileptogenic neoplasm with features of infiltrating astrocytoma and ependymoma. Journal of neuropathology and experimental neurology 2005 Oct; 64: 875-81

43 Thom M, Toma A, An S, Martinian L, Hadjivassiliou G, Ratilal B, et al. One hundred and one dysembryoplastic neuroepithelial tumors: an adult epilepsy series with immunohistochemical, molecular genetic, and clinical correlations and a review of the literature. Journal of neuropathology and experimental neurology 2011 Oct; 70: 859-78

44 Capper D, Jones DTW, Sill M, Hovestadt V, Schrimpf D, Sturm D, et al. DNA methylationbased classification of central nervous system tumours. Nature 2018 Mar 22; 555: 469-74

45 Qaddoumi I, Orisme W, Wen J, Santiago T, Gupta K, Dalton JD, et al. Genetic alterations in uncommon low-grade neuroepithelial tumors: BRAF, FGFR1, and MYB mutations occur at high frequency and align with morphology. Acta neuropathologica 2016 Jun; 131: 833-45

46 Aronica E, Crino PB. Epilepsy related to developmental tumors and malformations of cortical development. Neurotherapeutics : the journal of the American Society for Experimental NeuroTherapeutics 2014 Apr; 11: 251-68

47 de Groot M, Reijneveld JC, Aronica E, Heimans JJ. Epilepsy in patients with a brain tumour: focal epilepsy requires focused treatment. Brain : a journal of neurology 2012 Apr; 135: 1002-16

This article is protected by copyright. All rights reserved. 